\title{
Tumor-targeted efficiency of shRNA vector harboring chimera hTERT/U6 promoter
}

\author{
PENGHUI ZHANG ${ }^{1}$, YAQIN CHEN ${ }^{2}$, XIXIN JIANG ${ }^{2}$, ZHIGUANG TU $^{2}$ and LIN ZOU ${ }^{1}$ \\ ${ }^{1}$ Children's Hospital, Chongqing Medical University, Chongqing 400014; ${ }^{2}$ Faculty of Laboratory Medicine, \\ Key Laboratory of Laboratory Medical Diagnostics of Ministry of Education, \\ Chongqing Medical University, Chongqing 400016, P.R. China
}

Received December 10, 2009; Accepted February 8, 2010

DOI: $10.3892 /$ or_00000765

\begin{abstract}
Telomerase is closely related to tumor, and hTERT is the rate-limiting factor for telomerase activity. The transcription and expression of hTERT is determined by hTERT promoter, which has the ability of anchoring telomerase positive cells. RNA interference (RNAi) has been potentially used in the functional genomics and gene therapy recently. However, the limitations of RNAi uncertain interference and safety hamper its wide applications. To overcome these limitations, we constructed shRNA vectors harboring either U6 promoter or chimera hTERT/U6 promoter aiming at EGFP and hTERT genes (shRNA-EGFP-U6, shRNA-EGFPhTERT/U6, shRNA-hTERT-U6 and shRNA-hTERThTERT/U6), to suppress the expression of GFP and hTERT in telomerase negative human normal fibroblast HELF cells and telomerase positive human hepatocarcinoma SMMC7721 and HepG2 cells, respectively. HELF-EGFP and SMMC-7721-EGFP cells stably expressing EGFP or hTERT were constructed. GFP expression was inhibited in both HELF-EGFP and SMMC-7721-EGFP cells expressing shRNA-EGFP-U6. Further results showed that GFP expression was suppressed only in telomerase positive SMMC-7721 cells and HepG2 cells, but not in telomerase negative HELF cells expressing shRNA-EGFP-hTERT/U6. Further results found that hTERT expression was effectively inhibited from liver cancer cells expressing shRNA-hTERT-U6 or shRNAhTERT-hTERT/U6 both in vitro and in vivo. Our study illustrates the tumor-targeted efficiency of shRNA vectors harboring chimera hTERT/U6 promoter in telomerase positive cells, which will benefit tumor therapy.
\end{abstract}

Correspondence to: Dr Lin Zou, Center for Clinical Molecular Medicine, 136 Zhongshan Er Rd., Children's Hospital, Chongqing Medical University, Chongqing 400014, P.R. China

E-mail: zoulin74@hotmail.com

Key words: telomerase, human telomerase reverse transcriptase, short hairpin RNA, tumor-targeted, promoter

\section{Introduction}

Telomeres, the repeat short sequences of the end structure of chromosomes, maintain the normal length and function of chromosomes (1). In human somatic cells, telomeres typically consist of more than 1000 tandem repeats of nucleotides (CCCTAA in one strand of DNA and TTAGGG in the other) and associated proteins (1). These repeats are gradually lost with cellular replication and aging, owing to the inability of DNA polymerase to fully replicate the $3^{\prime}$ end of DNA (2). The attrition of repeats eventually shortens telomeres critically; the result is arrested proliferation and senescence, shortened life span, apoptosis, or genomic instability of the cell (3). Many investigations have shown that telomeres and telomerase are central to the biology of cancer, stem cells, and aging.

Maintenance of the integrity of telomeres requires the telomerase ribonucleoprotein complex, which consists of telomerase reverse transcriptase (TERT) and its integral RNA template (TERC), in addition to other proteins (4). Telomerase activity plays a key role in the normal function of telomeres. Telomerase is a ribonucleoprotein (RNP) enzyme that consists of an RNA moiety and several protein subunits (1). Among them, hTERT, also called the catalytic subunit of telomerase, is the rate-limiting factor of telomerase activity (5). Some studies have suggested that the transcription of hTERT represents the key steps in telomerase gene expression. hTERT transcription has been implied in cellular aging, immortalization, and transformation, which was determined by hTERT promoter. hTERT promoter was successfully cloned, characterized and function in 1999 (6), which could be detected in telomerase positive cells and tumors, but not in telomerase negative and normal cells. hTERT promoter was then broadly applied in the tumor biological therapy areas. Many investigations have showed that hTERT promoter driven different genes treated various kinds of telomerase positive tumors but not the telomerase negative tumors $(7,8)$, illustrating the tumor-targeted efficiency of hTERT promoter.

RNA interference (RNAi) is a natural process through which expression of a purpose gene can be knocked down theoretically with high specificity and selectivity (9). In recent years, RNAi has been potentially used in numerous research areas, including functional genomics, signal transduction and 
gene therapy (10). The applications of RNAi can be mediated through two types of molecules: the chemically synthesized double-stranded small interfering RNA (siRNA) or vector based short hairpin RNA (shRNA) (11). Due to fewer offtarget and more powerful effects produced by shRNA than by siRNA, the application of shRNA is more widely used than siRNA in the multiple areas (12). shRNAs are synthesized in the nucleus of cells, further processed and transported to the cytoplasm, and then incorporated into the RNA-induced silencing complex (RISC) for the activity (9). Although shRNA could knock down the expression of mammalian genes efficiently through selectively silenced the RNAi sequences, the shortcomings of shRNA include uncertain interference, diverse inhibition efficacy and safety of shRNA (13). Therefore, these disadvantages of shRNA hamper the applications of RNAi in further gene therapy and clinical research.

To overcome the limitations of shRNA application, we hypothesized that hTERT promoter could anchor telomerase positive tumor cells, together with U6 to form the efficient chimera hTERT/U6 promoter of shRNA, to suppress the purpose gene expression, and further inhibit the gene functions.

\section{Materials and methods}

Construction of multiple vectors. shRNA-vectors harboring either U6 promoter alone or chimera hTERT/U6 promoter for EGFP and hTERT genes, including shRNA-EGFP-U6, shRNA-EGFP-hTERT/U6, shRNA-hTERT-U6 and shRNAhTERT-hTERT/U6 were constructed. Briefly, shRNA-EGFPU6 and shRNA-hTERT-U6 were constructed as described (14). The transcript templates were: EGFP, 5'-CAGCCACA ACGTCTAATATCAT-3' (forward); 5'-AAGAACG GCA TCAAGGTGAACTT-3' (reverse); hTERT, 5'-TCGA CAGACACCAAGAAGCACGGAGAACTTCTTGGTG TTC-3' (forward); 5'-TAGAAAAAAGGAACACCAAGA AGTTCATCCGAAGATGAACTTCTTGGTGTCCTG-3' (reverse). Then shRNA-EGFP-hTERT/U6 and shRNAhTERT-hTERT/U6 were constructed based on shRNAEGFP-U6 and shRNA-hTERT-U6, respectively. The sequence of hTERT promoter was amplified from hTERT core sequence (6) by PCR, with KpnI and EcoRI enzyme sites at the end of 5'-flanking region. The primer sequence of hTERT core sequence was: 5'-AGGTACCACAGACGCCCAGGA CCGCGC-3' (forward); 5'-AGGAATTCGGCCAGGGCT TCCCACGTG-3' (reverse). All the PCR product of hTERT promoter, shRNA-EGFP-U6 and shRNA-hTERT-U6 were digested with KpnI and EcoRI (Takara, Otsu, Shiga, Japan), then ligated with T4 DNA ligase (Takara) and screened. All the vectors were identified by enzyme digestion and DNA sequencing.

Cell culture and screening. HELF and HepG2 cells were obtained from the American Type Culture Collection (Rockville, MD, USA), SMMC-7721 cells were obtained from Shanghai Tissue Cell Bank, which were maintained in Dulbecco's modified Eagle's medium (DMEM) (Gibco-BRL, Gaithersburg, MD, USA) supplemented with $10 \%$ FBS and antibiotics at $37^{\circ} \mathrm{C}$ in an atmosphere of $5 \% \mathrm{CO}_{2}$ and $10 \%$ charcoal-dextran-treated fetal calf serum (HyClone, Logan,
UT, USA). For generation of stable cell lines, HELF, SMMC7721 and HepG2 cells were transfected with $3 \mu \mathrm{g}$ of pcDNA3 containing EGFP, shRNA-EGFP-U6, shRNA-EGFPhTERT/U6, shRNA-hTERT-U6, shRNA-hTERT-hTERT/U6, respectively using Lipofectamine 2000 (Invitrogen, Carlsbad, CA, USA) according to the manufacturer's instructions. Stable cell expression clones were selected and maintained in G418 $(300 \mu \mathrm{g} / \mathrm{ml})$, and generated HELF-EGFP, 7721-EGFP, HepG2EGFP, 7721-shRNA-hTERT-U6, 7721-shRNA-hTERThTERT/U6, and 7721-U6 cells.

$R T-P C R$. Total RNAs were extracted from tissues with TRIzol (Invitrogen) according to the manufacturer's instructions. Reverse transcription of purified RNA was performed using oligo (dT) primer and superscript II reverse transcriptase (Invitrogen). Then quantification of the genes was performed by PCR. Expression values were normalized to those obtained with control GAPDH gene. The primer pairs were: EGFP, 5'-CGTCCATGCCGAGAGTGATC-3' (forward), 5'-CCG ACAACCACTACCTGAGC-3' (reverse) ; GAPDH, 5'-CCA TGGAGAAGGCTGGGG-3' (forward), 5'-CAAAGTTGT CATGGATGACC-3' (reverse); hTERT, 5'-CGGAAGAGT GTCTGGAGCAA-3' (forward), 5'-GGATGAAGCGGA GTCTGGA-3' (reverse). Reaction buffer in $50 \mu \mathrm{l}$ volume included $10 \mathrm{X}$ buffer, $1 \mu \mathrm{g}$ cDNA, $200 \mathrm{nM} \mathrm{MgCl}$, Taq polymerase $2.5 \mathrm{U}$ and $20 \mathrm{nM}$ dNTP. The PCR amplification was 35 cycles with each of denaturing at $94^{\circ} \mathrm{C}$ for $60 \mathrm{sec}$, annealing at $60^{\circ} \mathrm{C}$ for $30 \mathrm{sec}$ and extension at $72^{\circ} \mathrm{C}$ for $60 \mathrm{sec}$. The PCR product was electrophoresis in $2 \%$ agarose and imaged with image photograph (Bio-Rad, CA, USA). The optical density (OD) value of each band was quantified and calculated by using Scion Image software.

Western blotting. Cells or tissue proteins were lysed on ice by RIPA buffer (50 mM Tris-HCl, pH 7.5; $150 \mathrm{mM} \mathrm{NaCl}$; $1 \%$ Nonidet P-40; $0.1 \%$ SDS; and $0.5 \%$ sodium deoxycholate) and boiled for $10 \mathrm{~min}$ in sample buffer $(62.5 \mathrm{mM}$ Tris- $\mathrm{HCl}$, pH 6.8; $10 \%$ glycerol; $2 \%$ SDS; and $50 \mathrm{mM}$ dithiothreitol), as the previous study (15). Equivalent amounts of protein were subjected to SDS-PAGE electrophoresis and then electroblotted onto a polyvinylidene difluoride membrane. The membrane was incubated with primary antibodies against EGFP or hTERT (Santa Cruz, CA, USA), and then HRPconjugated secondary antibody, detected using the ECL Plus Western detecting kit (Amersham Pharmacia, Piscataway, NJ, USA). The OD value of each band was also quantified and calculated by using Scion Image software.

Immunofluorescence. Cells grown on coverglass were transfected with or without the plasmids. After $48 \mathrm{~h}$, cells were fixed by ice cold $4 \%$ PFA, washed and incubated with primary antibodies at $4{ }^{\circ} \mathrm{C}$ overnight, then with fluorophoreconjugated secondary antibodies, and observed with a DML-IL Fluorescence microscope (Leica Microsystems, Bensheim, Germany).

Animal experiment. For animal experiments, establishing liver cancer xenograft tumor model was as described previously (14). 7721-U6, 7721-shRNA-hTERT-U6 or 7721-shRNAhTERT-hTERT/U6 stable cells $\left(2.0 \times 10^{6}\right.$ cells in $0.5 \mathrm{ml}$ of 


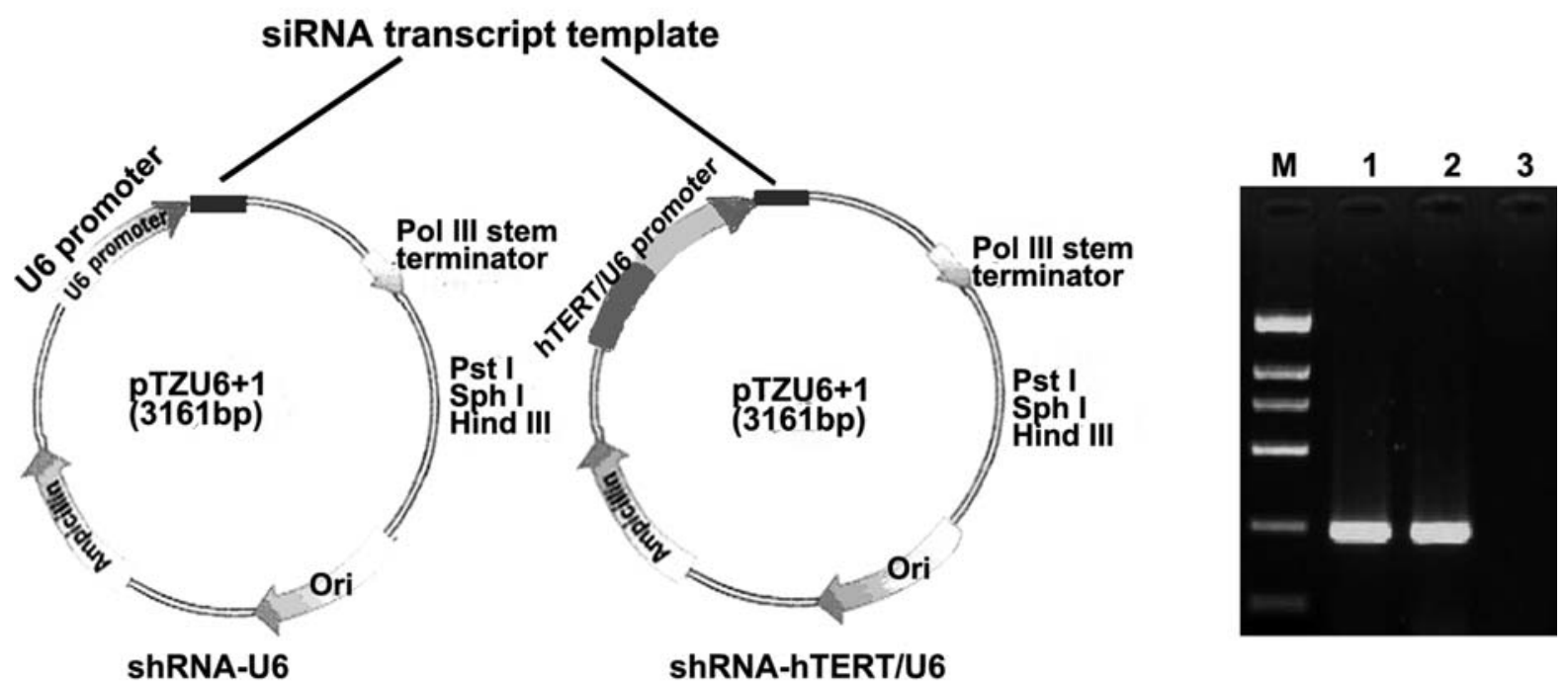

C
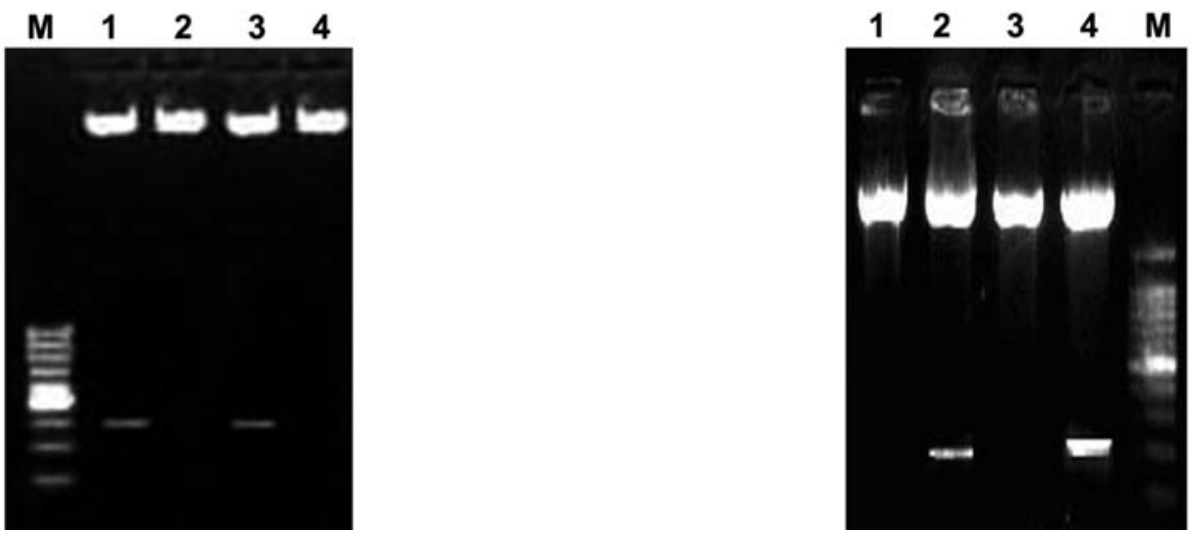

Figure 1. Successful construction of chimera hTERT/U6 promoter, shRNA-EGFP-U6, shRNA-EGFP-hTERT/U6, shRNA-hTERT-U6 and shRNA-hTERThTERT/U6 vectors. (A) The scheme of different vector construction. (B) PCR identification of hTERT core promoter. (C) Identification of shRNA-EGFP vectors. M, DNA Ladder Marker; lane 1, shRNA-EGFP-U6; lane 2, shRNA-EGFP-U6 digested with SalI and XbaI; lane 3, shRNA-EGFP-hTERT/U6; lane 4, shRNA-EGFP-hTERT/U6 digested with SalI and XbaI. (D) Enzyme digestion of shRNA-hTERT vectors. Lane 1, shRNA-hTERT-U6; lane 2, shRNA-hTERTU6 digested with SalI and XbaI; lane 3, shRNA-hTERT-hTERT/U6; lane 4, shRNA-hTERT-hTERT/U6 digested with SalI and XbaI; M, DNA Ladder Marker.

serum-free RPMI-1640 medium) were injected s.c. into the right back of 8-10-week-old male BALB/c nude mice (ten mice for each group), and the tumor growth was monitored using electronic calipers every other day (14). Mice were sacrificed by cervical dislocation the day after the final treatment. The tumors were removed and frozen rapidly, total RNA and protein were isolated from frozen tumors, and then detected the alteration of gene expression. The results represented are the mean value of three independent experiments. All the animal experiments were approved by the institutional and governmental animal review boards.

Statistical analysis. All measurements were performed in at least three independent experiments and mean \pm SE was obtained. Data were analyzed by Student's t-test (two-tailed) for comparison of independent means, with pooled estimates of common variances. For all tests, the criterion for statistical significance was taken as $\mathrm{P}<0.05$.

\section{Results}

Successful construction of shRNA vectors with U6 or chimera hTERT/U6 promoter and stable expressing cells. To understand the efficiency of RNAi, we constructed shRNA vectors aiming at EGFP and hTERT genes by using U6 promoter alone or chimera hTERT/U6 promoter, which is illustrated in Fig. 1A. To construct the vectors with chimera hTERT/U6 promoter, we amplified the core sequence from the hTERT promoter (Fig. 1B), and then ligated with shRNA-EGFP-U6 or shRNA-hTERT-U6 vector, identified with enzyme digestion (Fig. 1C and D). All the vectors were identified with DNA sequencing and the sequences were correct (data not shown). We then constructed the stable human hepatocarcinoma SMMC-7721 cells and human normal fibroblast HELF cells expressing EGFP gene with G418 screening, which are shown with immunofluorescence image in Fig. 2A. To further identify the expression of GFP in the 

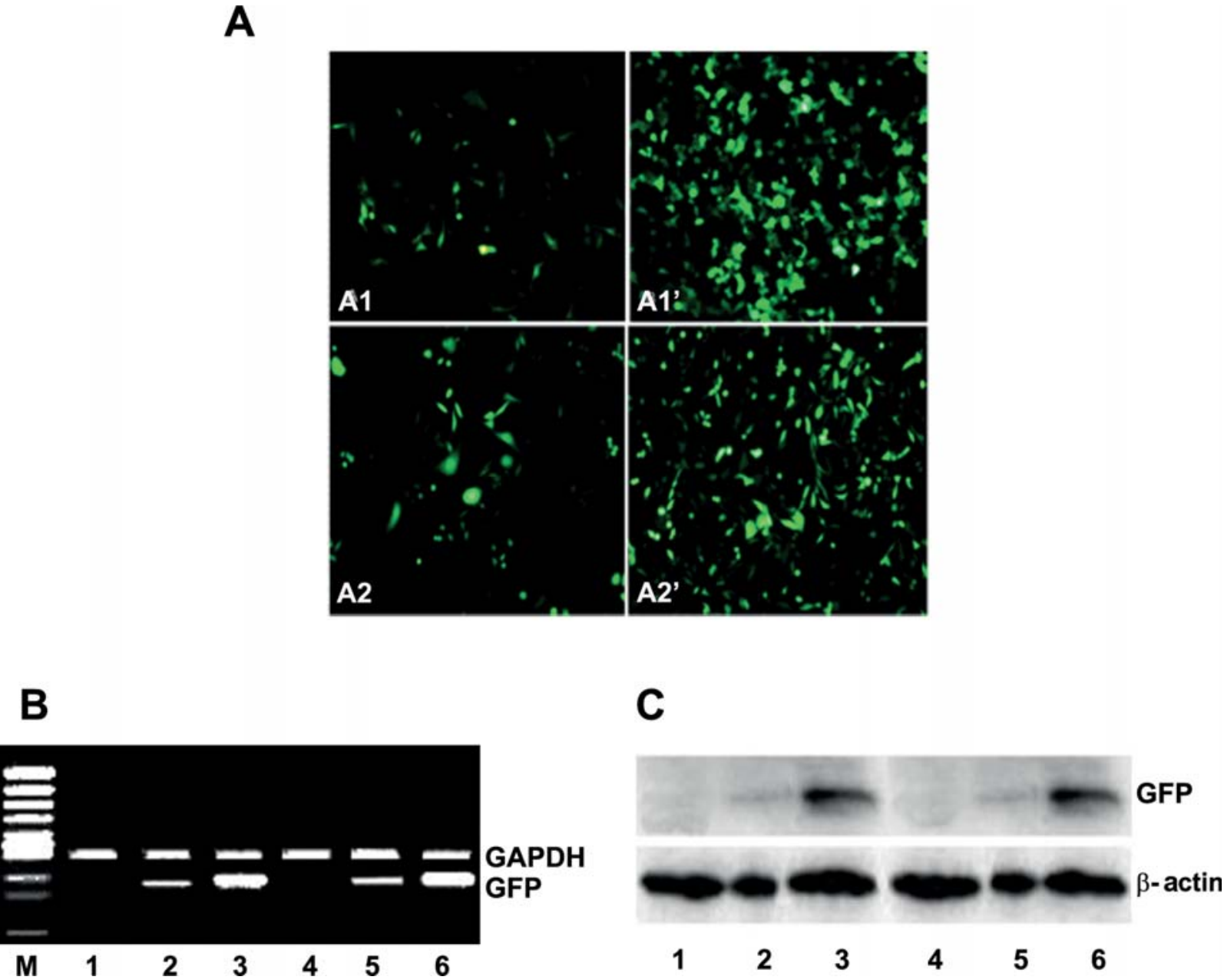

Figure 2. Successful establishment of stable HELF-EGFP cells and 7721-EGFP cells. (A) Immunofluorescence identification of GFP stable expression in SMMC-7721 and HELF cells. Human hepatocarcinoma SMMC-7721 cell and human normal fibroblast HELF cell transient expression of GFP (48 h); Human hepatocarcinoma SMMC-7721 cells stable expression of GFP by screening with $800 \mu \mathrm{g} / \mathrm{ml} \mathrm{G} 418$ for 10 days, then maintained at $300 \mu \mathrm{g} / \mathrm{ml} \mathrm{G} 418 \mathrm{culture}$ medium. A1, SMMC-7721 cells transient expression of EGFP; A1', SMMC-7721 cell stable expression of EGFP (7721-EGFP cells); A2, HELF cell transient expression of EGFP; A2', HELF cell stable expression of EGFP (HELF-EGFP cells). Scale bar, $40 \mu \mathrm{m}$. (B) RT-PCR identification of GFP expression in cells . (C) WB identification of GFP expression in cells. M, DNA Ladder Marker; lane 1, SMMC-7721 cells without transfection EGFP; lane 2, SMMC-7721 cells transient expression of EGFP; lane 3, 7721-EGFP stable cells; lane 4, HELF cells without transfection EGFP; lane 5, HELF cells transient expression of EGFP; lane 6, HELF-EGFP stable cells.

stable cells, we then used RT-PCR (Fig. 2B) and Western blot (Fig. 2C) to show much stronger GFP expression in stable cells than in transient cells, named as 7721-EGFP and HELF-EGFP cells. SMMC-7721, HepG2 and HELF cells stably expressing shRNA-EGFP-U6, shRNA-hTERT-U6, shRNA-EGFP-hTERT/U6 and shRNA-hTERT-hTERT/U6 were also constructed with similar strategy (data not shown), named as 7721-shRNA-EGFP-U6, HepG2-shRNA-EGFPhTERT/U6 cells, and HELF-shRNA-EGFP-U6.

shRNA-EGFP blocks GFP expression efficiently. To measure the RNAi efficiency of shRNA, we chose GFP as the purpose gene to detect the inhibition effect. All the data showed that the expression of GFP both in HELF-EGFP cells and 7721EGFP cells was remarkably reduced in cells stably expressed with shRNA-EGFP-U6 compared with cells expressed with empty vector, which showed consistently positive results from the immunofluorescence microscopy (Fig. 3A), RT-PCR (Fig. 3B) and Western blot (Fig. 3C) experiments.
GFP expression was selectively knocked down in telomerase positive cells by shRNA-EGFP with chimera hTERT/U6 promoter. To further investigate the blocking efficiency of shRNA-EGFP in telomerase positive cells, we applied telomerase positive SMMC-7721 cells and telomerase negative HELF cells to construct stable cells expressing shRNA-EGFPU6 and shRNA-EGFP-hTERT/U6, named as 7721-shRNAEGFP-U6 cells, 7721-shRNA-EGFP-hTERT/U6 cells, HELFshRNA-EGFP-U6 cells and HELF-shRNA-EGFP-hTERT/U6 cells. GFP expression was then detected in these cells with various assays. The results showed that the expression of GFP was remarkably suppressed both in 7721-shRNA-EGFP-U6 cells and HELF-shRNA-EGFP-U6 cells compared with 7721-EGFP-U6 cells and HELF-EGFP-U6 cells, respectively. Interestingly, the expression of GFP was inhibited only in 7721-shRNA-EGFP-hTERT/U6 cells, but not in HELFshRNA-EGFP-hTERT/U6 cells (Fig. 4A-C), indicating that GFP expression was selectively knocked down in telomerase positive cells by shRNA-EGFP with chimera hTERT/U6 promoter. 


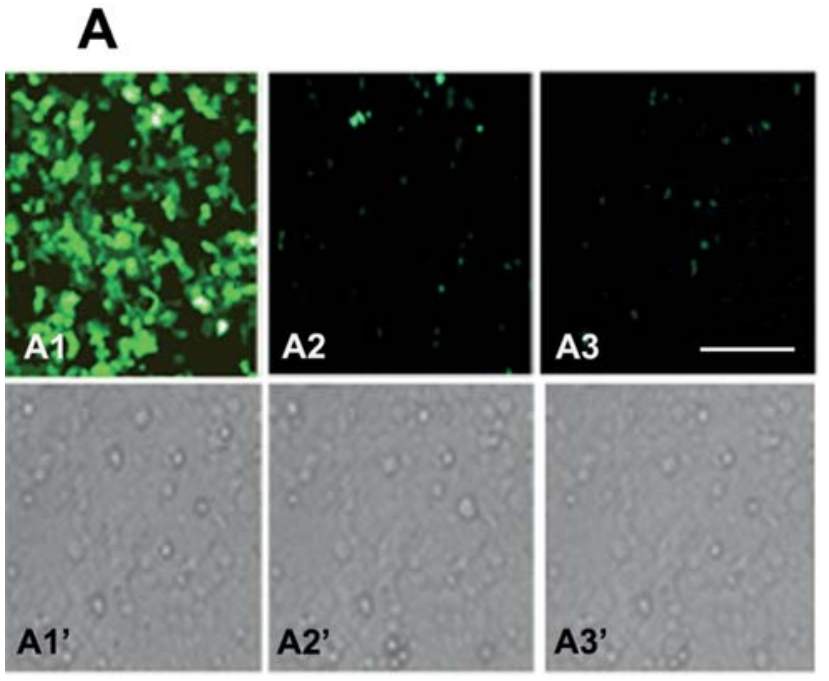

Figure 3. shRNA-EGFP blocks GFP expression in HELF-EGFP and 7721EGFP cells. (A) Immunofluorescence identification of GFP expression in different cells. A1, HELF-EGFP-U6 cells; A2, HELF-shRNA-EGFP-U6 cells; A3, 7721- shRNA-EGFP-U6 cells; A1'-A3', the bright light view of A1-A3, respectively. Scale bar, $40 \mu \mathrm{m}$. (B) PCR identification of GFP expression in different cells. Lane 1, HELF-EGFP-U6 cells; lane 2, HELFshRNA-EGFP-U6 cells; lane 3, 7721-shRNA-EGFP-U6 cells. B', the statistical graph of GFP/GAPDH OD value of B. The numbers are mean \pm $\mathrm{SE}$, and the mean is the fold-value compared with that in HELF-EGFP-U6 cells. (C) Western blot identification of GFP expression in different cells. Lane 1, HELF-EGFP-U6 cells; lane 2, HELF-shRNA-EGFP-U6 cells; lane 3, 7721-shRNA-EGFP-U6 cells. $C^{\prime}$, the statistical graph of GFP/B-actin OD value of $\mathrm{C}$. The number is mean $\pm \mathrm{SE}$, and the mean is the fold-value compared with that in HELF-EGFP-U6 cells.
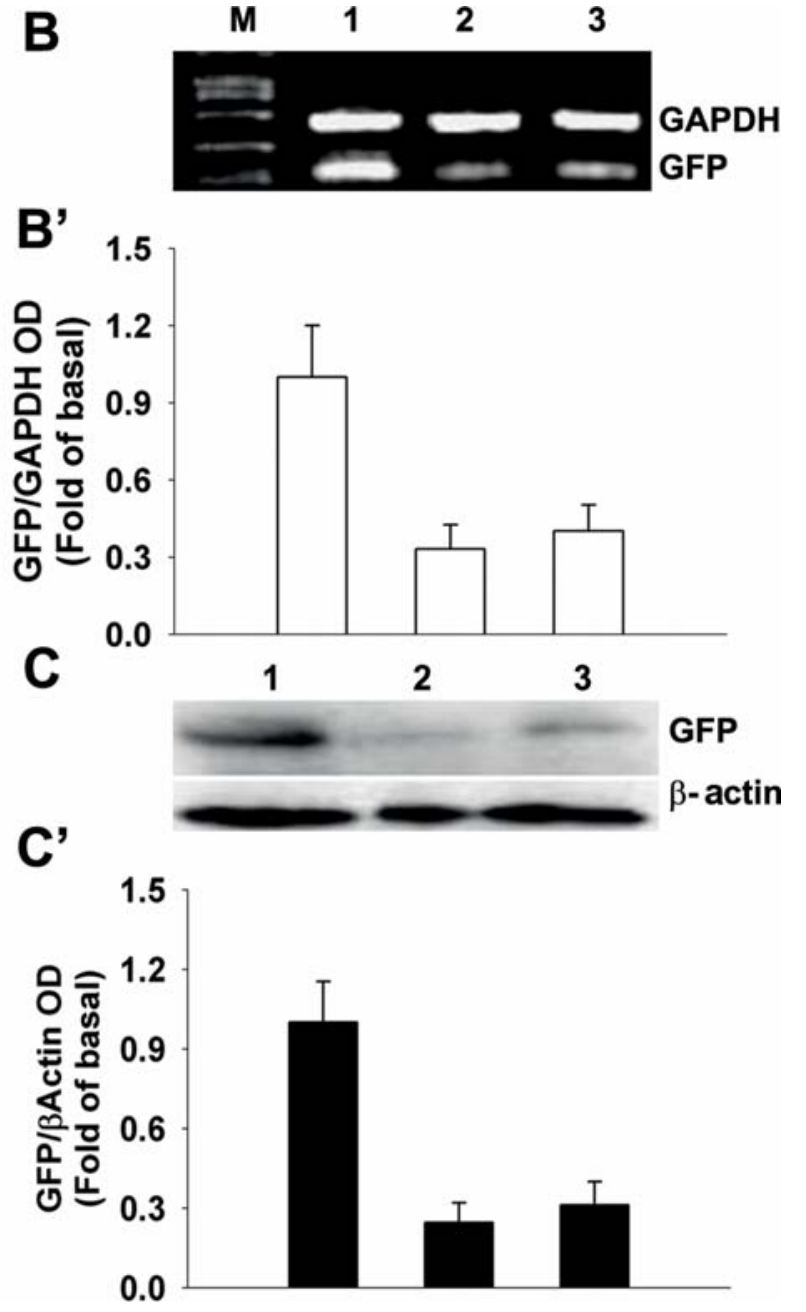

B

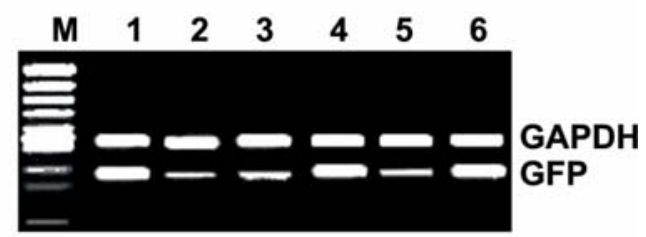

C

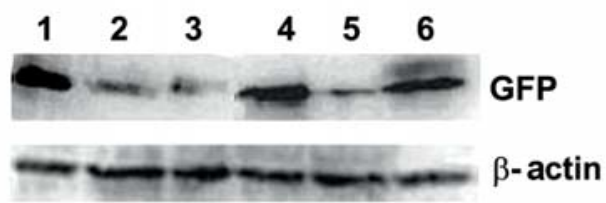

Figure 4. Inhibited GFP expression in 7721-shRNA-EGFP-hTERT/U6 cells but not in HELF-shRNA-EGFP-hTERT/U6 cells. (A) Immunofluorescence identification of GFP expression in different cells. A1, HELF-EGFP-U6 cells; A2, 7721-EGFP-U6 cells; A3, HELF-shRNA-EGFP-U6 cells; A4, 7721-shRNA-EGFP-U6 cells; A5, HELF-shRNA-EGFP-hTERT/U6 cells; A6, 7721-shRNA-EGFP-hTERT/U6 cells. (B) RT-PCR identification of GFP expression in different cells. (C) Western blot identification of GFP expression in different cells. M, Marker. Lane 1, 7721-EGFP-U6 cells; lane 2, 7721-shRNA-EGFP-U6 cells; lane 3, 7721-shRNA-EGFP-hTERT/U6 cells; lane 4, HELF-EGFP-U6 cells; lane 5, HELF-shRNA-EGFP-U6 cells; lane 6, HELF-shRNA-EGFP-hTERT/U6 cells. 

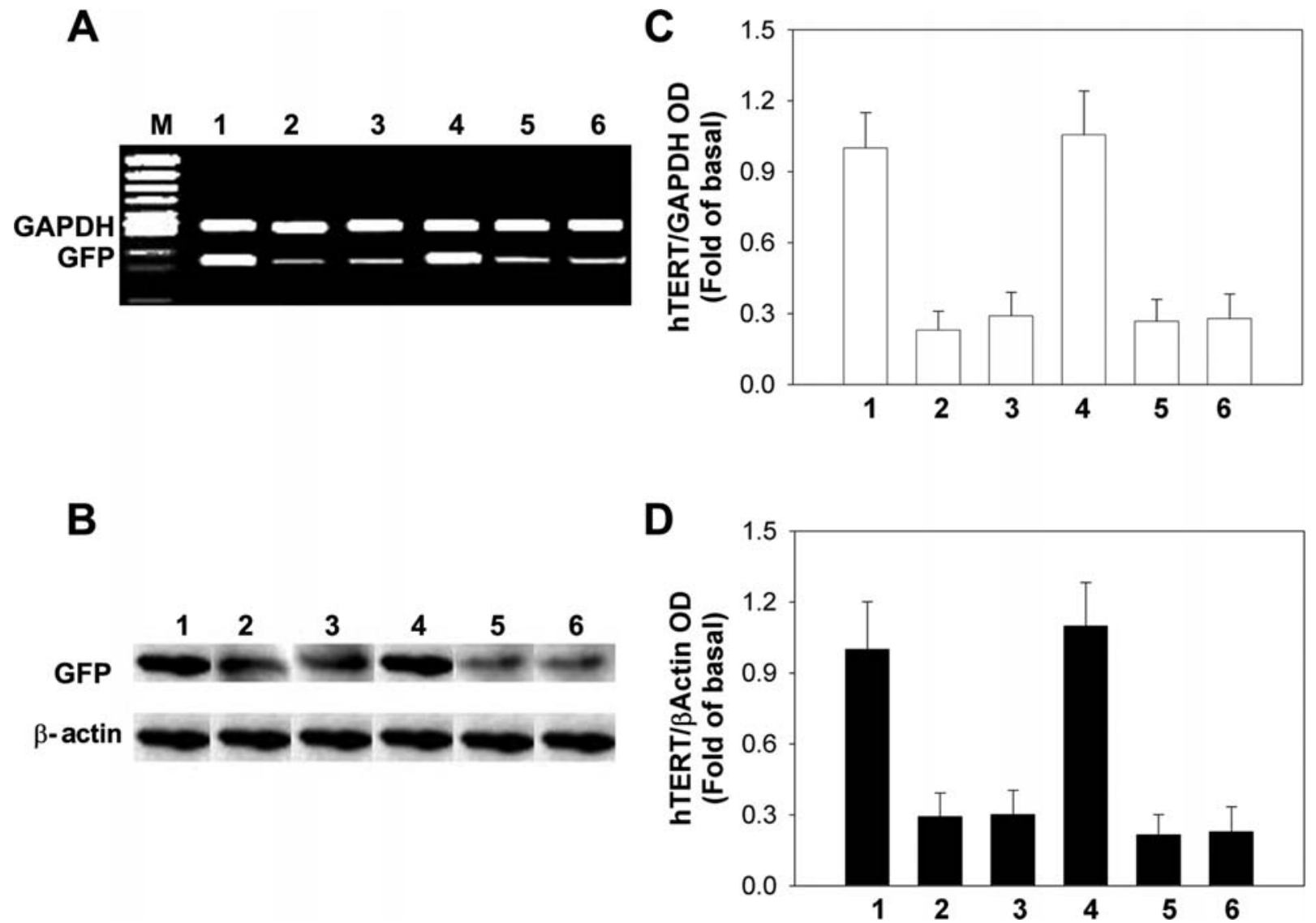

Figure 5. Suppressed hTERT expression by shRNA-hTERT with U6 or U6/hTERT promoter in liver cancer SMMC-7721 and HepG2 cells. (A) hTERT expression by RT-PCR identification in different cells. (B) hTERT expression by Western blot in different cells. (C) The statistical graph of hTERT/GAPDH OD value of $A$. The number is mean $\pm \mathrm{SE}$, and means is the fold-value compared with that in 7721-U6 cells. (D) The statistical graph of hTERT/B-actin OD value of $\mathrm{B}$. The number is mean $\pm \mathrm{SE}$, and means is the fold-value compared with that in 7721-U6 cells. M, Marker; 1, 7721-U6 cells; 2, 7721-shRNAhTERT-U6 cells; 3, 7721-shRNA-hTERT-hTERT/U6 cells; 4, HepG2-U6 cells; 5, HepG2-shRNA-hTERT-U6 cells; 6, HepG2-shRNA-hTERT-hTERT/U6 cells.

hTERT expression is efficiently suppressed by shRNA-hTERT with U6 or hTERT/U6 promoter in liver cancer cells in vitro and in vivo. To understand the expression of hTERT in diverse cells expressing shRNA-hTERT with U6 or hTERT/U6 promoter in liver cancer cells, we generated shRNA vectors aiming at hTERT gene and harboring U6 or hTERT/U6 promoter, then constructed liver cancer SMMC-7721 and HepG2 cells stably expressing these shRNA-hTERT vectors. The expression of hTERT was detected in these stable liver cancer cells. Both the results from RT-PCR (Fig. 5A and C) and Western blot (Fig. 5B and D) assays showed that hTERT expression was obviously reduced in 7721-shRNA-hTERTU6 cells, 7721-shRNA-hTERT-hTERT/U6 cells, HepG2shRNA-hTERT-U6 cells and HepG2-shRNA-hTERThTERT/U6 cells, comparing with 7721-U6 cells and HepG2U6 cells. These data showed inhibition efficiency of shRNAhTERT in these liver cancer cells in vitro.

We then detected the hTERT expression by shRNAhTERT in an animal model. By using the xenograft liver cancer model, we subcutaneously inoculated the same amount of liver cancer 7721-shRNA-hTERT-U6 or 7721-shRNAhTERT-hTERT/U6 stable cells into BALB/c nude mice, 7721-U6 stable cells as the control, found that the tumor grew much slower both in mice injected with 7721-shRNAhTERT-hTERT/U6 cells and 7721-shRNA-hTERT-U6 cells compared with 7721-U6 cells (Fig. 6A). The tumor volume of mice in these previous two groups was much smaller than in mice treated with 7721-U6 cells in post-injected 28 days (Fig. 6B). The expression of hTERT was blocked in the tumor tissues of mice injected with 7721-shRNA-hTERThTERT/U6 cells and 7721-shRNA-hTERT-U6 cells compared with 7721-U6 cells, based on the results from RTPCR (Fig. 6C) and Western blot (Fig. 6D), further confirming the inhibition efficiency of shRNA-hTERT in liver cancer cells in vivo.

\section{Discussion}

RNA interference (RNAi) is the recent highlight of discovery in cell biology, being able to knock down the expression of a purpose gene with high specificity and selectivity (9). RNAi presents a valuable tool for personalized cancer therapy and can also be applied in combination with immune modulating agents or small molecules to improve the efficacy of cancer and other disease treatment (16-19). The applications of RNAi could be mediated through siRNA or shRNA (11). Herein, we adopted shRNA strategy of RNAi, aiming at EGFP and hTERT genes, to silence the intrinsic expression of these genes. Our results testified that the expression of GFP was inhibited efficiently in HELF-EGFP cells, 7721-EGFP and 
A

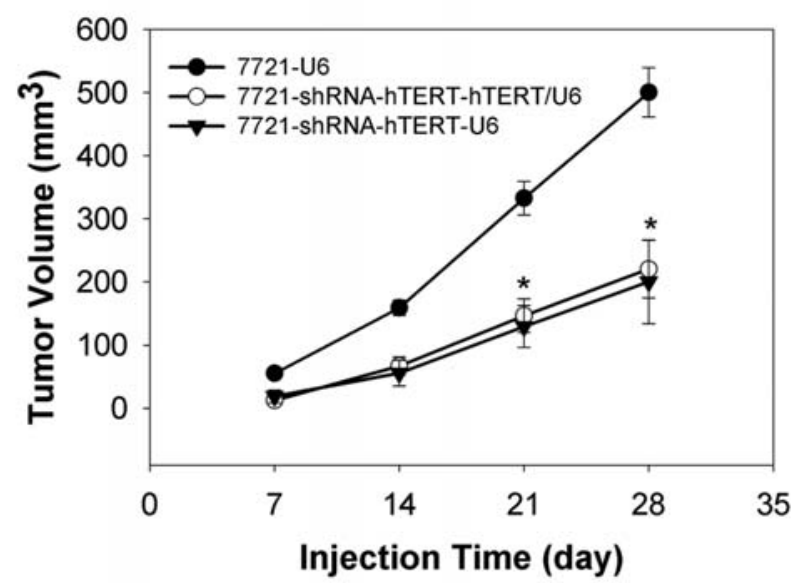

C

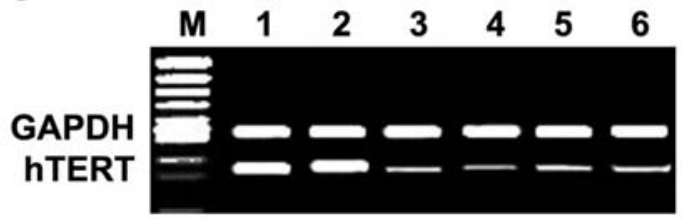

C'

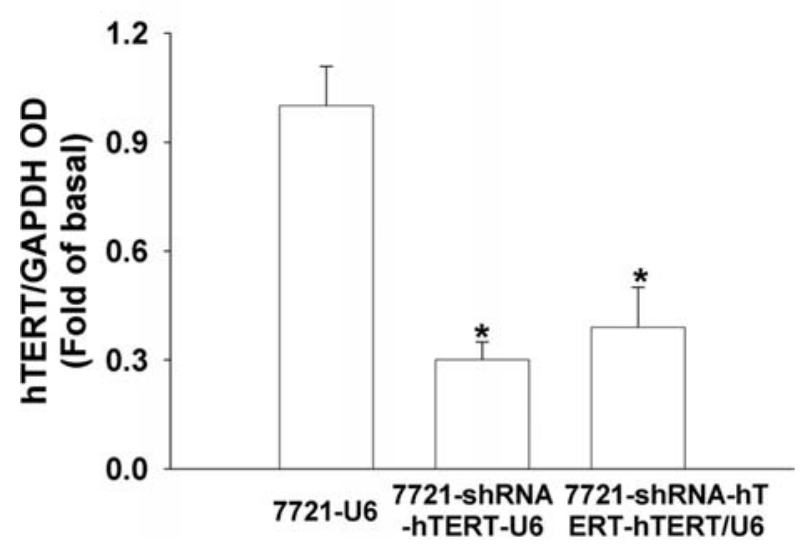

B

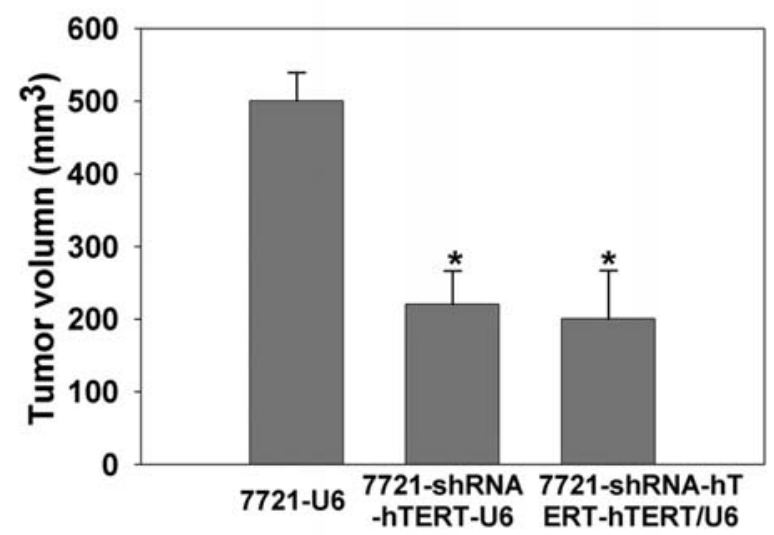

D

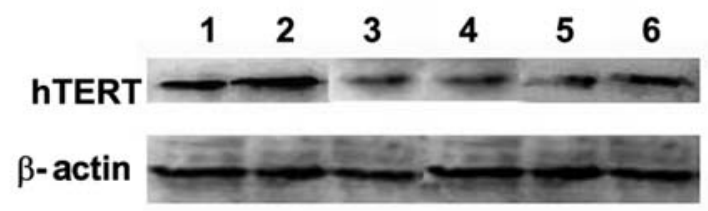

D'

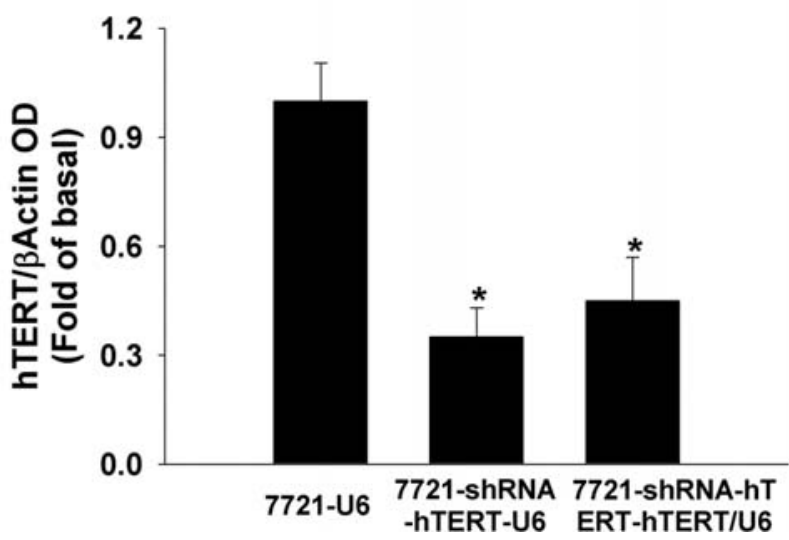

Figure 6. Suppressed hTERT expression in xenograft liver cancer cells by shRNA-hTERT with U6 or U6/hTERT promoter. (A) Dynamic tumor volume of mice in different groups. ${ }^{*} \mathrm{P}<0.05$ vs. 7721 -U6 group. (B) Tumor volume of mice in different groups at post-injected 28 days. The groups were mice treated with the same amount of 7721-shRNA-hTERT-hTERT/U6 cells, 7721-shRNA-hTERT-U6 cells or 7721-U6 cells, respectively. *P<0.05 vs. 7721 -U6 group. (C) hTERT expression in different treated mouse tumors by RT-PCR. (C') The statistical graph of hTERT/GAPDH OD value of C. The number is mean \pm SE, and means is the fold-value compared with that in 7721-U6 cells. ${ }^{*} \mathrm{P}<0.05$ vs. 7721-U6 group. (D) hTERT expression in different treated mouse tumors by Western blot. (D') The statistical graph of hTERT/ß-actin OD value of $\mathrm{D}$. The number is mean $\pm \mathrm{SE}$, and means is the fold value compared with that in $7721-\mathrm{U} 6$ cells. ${ }^{*} \mathrm{P}<0.05$ vs. 7721-U6 group. Lanes 1 and 2, tumors of mice treated with 7721-U6 cells; lanes 3 and 4, tumors of mice treated with 7721-shRNA-hTERT-U6 cells; lanes 5 and 6 , tumors of mice treated with 7721-shRNA-hTERT-hTERT/U6 cells.

HepG2-EGFP cells by expressing EGFP-shRNA, indicating the correct strategy and effective sequences of the selective gene for shRNA. Also, hTERT expression was knocked down in the telomerase positive liver cancer cells by hTERTshRNA both in vitro and in vivo, further indicating that shRNA strategy and the selective gene sequences for RNAi were effective.

Conceptually, RNAi could block down the targeted gene in a specific and selective manner. However, when RNAi mediate the gene expression, it could also trigger unintended effects ('off-target effects') (20), nonspecific interferon (INF) response in immunology system (21), undesirable side effects by cross-reactions with the endogenous miRNA pathway (22), and numerous toxic side effects (23). To overcome these limitations, lower concentrations of siRNA and shRNA strategy were previously applied $(20,23)$. In combination with the hTERT promoter in telomerase positive cells for tumor gene therapy $(7,8)$, we constructed shRNAEGFP vectors harboring U6 promoter alone or hTERT/U6 chimera promoter, stably expressed in telomerase positive and negative cells, and found that GFP expression could be blocked by shRNA-EGFP vectors with U6 promoter both in 
telomerase positive cells (SMMC-7721 cells) and telomerase negative cells (HELF cells). However, GFP expression could only be blocked by EGFP-shRNA vectors with hTERT/U6 promoter in telomerase positive cells (SMMC-7721 cells), other than telomerase negative cells (HELF cells), indicating the tumor-targeted effect of shRNA vectors with chimera U6/hTERT promoter. The fact that hTERT-shRNA with hTERT/U6 promoter could efficiently suppress the expression of hTERT in liver cancer cells both in vitro and in vivo further validates the outcome of tumor-targeted effect of shRNA vectors with chimera hTERT/U6 promoter. This should be confirmed by various tumor types in the future.

RNAi has been applied in gene function investigations (24) and genome-wide library screens (25) in basic research. In clinical area, RNAi-based therapeutics have been preliminarily applied in eye diseases (26), viral infection (27) and cancers (28). The most concern in RNAi application is the few off-target and side effects (9). Since more than 95\% cancers are telomerase positive, optimized shRNA with hTERT promoter could be a potential approach for high potency and sustainable effects of knocking down the purpose genes effectively, which will benefit tumor therapeutic methods with fewer side effects.

\section{Acknowledgements}

We would like to thank Dr Ailong Huang from Chongqing Medical University for the kind gift of pTZU6+1 empty vector, and $\mathrm{Dr}$ K.J. Wu for the kind gift of hTERT promoter. This work was supported by National Natural Science Foundation of China (30400166, 30400539, 30871103, 90919013), New Century Talented Program from Education Ministry, Chongqing Natural Scientific Fund (CQST20082207).

\section{References}

1. Greider CW: Telomere length regulation. Annu Rev Biochem 65: 337-365, 1996.

2. Cervantes RB and Lundblad V: Mechanisms of chromosomeend protection. Curr Opin Cell Biol 14: 351-356, 2002.

3. Shay JW and Wright WE: Senescence and immortalization: role of telomeres and telomerase. Carcinogenesis 26: 867-874, 2005.

4. Feng J, Funk WD, Wang SS, et al: The RNA component of human telomerase. Science 269: 1236-1241, 1995.

5. Nakamura TM, Morin GB, Chapman KB, et al: Telomerase catalytic subunit homologs from fission yeast and human. Science 277: 955-959, 1997.

6. Wu KJ, Grandori C, Amacker M, et al: Direct activation of TERT transcription by c-myc. Nat Genet 21: 220-224, 1999.
7. Kim SH, Chung HK, Kang JH, et al: Tumor-targeted radionuclide imaging and therapy based on human sodium iodide symporter gene driven by a modified telomerase reverse transcriptase promoter. Hum Gene Ther 19: 951-957, 2008

8. Shay JW and Wright WE: Telomerase: A target for cancer therapeutics. Cancer Cell 2: 257-265, 2002.

9. Ichim TE, Li M, Qian H, et al: RNA interference: a potent tool for gene-specific therapeutics. Am J Transplant 4: 1227-1236, 2004.

10. Hannon GJ and Rossi JJ: Unlocking the potential of the human genome with RNA interference. Nature 431: 371-378, 2004.

11. Dykxhoorn DM, Novina CD and Sharp PA: Killing the messenger: short RNAs that silence gene expression. Nat Rev Mol Cell Biol 4: 457-467, 2003.

12. Meister G and Tuschl T: Mechanisms of gene silencing by double-stranded RNA. Nature 431: 343-349, 2004.

13. An DS, Xie Y, Mao SH, et al: Efficient lentiviral vectors for short hairpin RNA delivery into human cells. Hum Gene Ther 14: 1207-1212, 2003.

14. Zhang PH, Zou L and Tu ZG: RNAi-hTERT inhibition hepatocellular carcinoma cell proliferation via decreasing telomerase activity. J Surg Res 131: 143-149, 2006.

15. Nishi H, Nakada T, Kyo S, et al: Hypoxia-inducible factor 1 mediates upregulation of telomerase (hTERT). Mol Cell Biol 24: 6076-6083, 2004

16. Collins I and Workman P: New approaches to molecular cancer therapeutics. Nat Chem Biol 2: 689-700, 2006.

17. Soifer HS, Rossi JJ and Sætrom P: MicroRNAs in disease and potential therapeutic applications. Mol Ther 15: 2070-2079, 2007.

18. Hauf S, Cole RW, LaTerra S, et al: The small molecule Hesperadin reveals a role for Aurora B in correcting kinetochoremicrotubule attachment and in maintaining the spindle assembly checkpoint. J Cell Biol 161: 281-294, 2003.

19. Chen Y, Stamatoyannopoulos G and Song CZ: Down-regulation of CXCR4 by inducible small interfering RNA inhibits breast cancer cell invasion in vitro. Cancer Res 63: 4801-4804, 2003.

20. Jackson AL and Linsley PS: Noise amidst the silence: off-target effects of siRNAs? Trends Genet 20: 521-524, 2004.

21. Sledz CA, Holko M, de Veer MJ, et al: Activation of the interferon system by short-interfering RNAs. Nat Cell Biol 5: 834-839, 2003

22. Golzio M, Mazzolini L, Ledoux A, et al: In vivo gene silencing in solid tumors by targeted electrically mediated siRNA delivery. Gene Ther 14: 752-759, 2007.

23. Fedorov Y, Anderson EM, Birmingham A, et al: Off-target effects by siRNA can induce toxic phenotype. RNA 12: 1188-1196, 2006.

24. Dorsett $Y$ and Tuschl T: siRNAs: applications in functional genomics and potential as therapeutics. Nat Rev Drug Discov 3: 318-329, 2004

25. Paddison PJ, Silva JM, Conklin DS, et al: A resource for largescale RNA-interference-based screens in mammals. Nature 428: 427-431, 2004.

26. Kim B, Tang Q, Biswas PS, et al: Inhibition of ocular angiogenesis by siRNA targeting vascular endothelial growth factor pathway genes: therapeutic strategy for herpetic stromal keratitis. Am J Pathol 165: 2177-2185, 2004.

27. Ding SW and Voinnet O: Antiviral immunity directed by small RNAs. Cell 130: 413-426, 2007.

28. Takei Y, Kadomatsu K, Yuzawa Y, et al: A small interfering RNA targeting vascular endothelial growth factor as cancer therapeutics. Cancer Res 64: 3365-3370, 2004. 